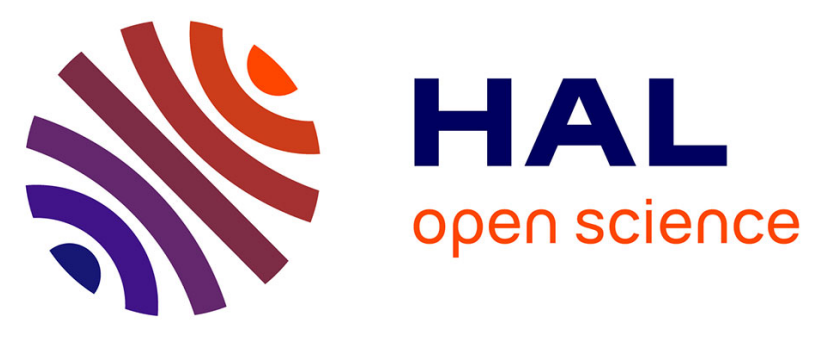

\title{
Immunological and clinical effects of low-dose interleukin-2 across 11 autoimmune diseases in a single, open clinical trial
}

Michelle Rosenzwajg, Roberta Lorenzon, Patrice Cacoub, Hang Phuong Pham, Fabien Pitoiset, Karim El Soufi, Claire Ribet, Claude Bernard, Selim Aractingi, Beatrice Banneville, et al.

\section{To cite this version:}

Michelle Rosenzwajg, Roberta Lorenzon, Patrice Cacoub, Hang Phuong Pham, Fabien Pitoiset, et al.. Immunological and clinical effects of low-dose interleukin-2 across 11 autoimmune diseases in a single, open clinical trial. Annals of the Rheumatic Diseases, 2018, pp.annrheumdis-2018-214229. hal-01960735

\section{HAL Id: hal-01960735 \\ https: / hal.sorbonne-universite.fr/hal-01960735}

Submitted on 8 Jan 2019

HAL is a multi-disciplinary open access archive for the deposit and dissemination of scientific research documents, whether they are published or not. The documents may come from teaching and research institutions in France or abroad, or from public or private research centers.
L'archive ouverte pluridisciplinaire HAL, est destinée au dépôt et à la diffusion de documents scientifiques de niveau recherche, publiés ou non, émanant des établissements d'enseignement et de recherche français ou étrangers, des laboratoires publics ou privés. 


\title{
Immunological and clinical effects of low-dose interleukin-2 across 11 autoimmune diseases in a single, open clinical trial
}

\author{
Michelle Rosenzwajg, ${ }_{1}^{1,2}$ Roberta Lorenzon, ${ }^{1,2}$ Patrice Cacoub, ${ }^{1,2,3}$ \\ Hang Phuong Pham, ${ }^{4}$ Fabien Pitoiset, ${ }^{1,2}$ Karim El Soufi, ${ }^{1,2}$ Claire RIbet, ${ }^{1}$ \\ Claude Bernard, ${ }^{1,2}$ Selim Aractingi, ${ }^{5}$ Beatrice Banneville, ${ }^{6}$ Laurent Beaugerie, ${ }^{7}$ \\ Francis Berenbaum, ${ }^{8}$ Julien Champey, ${ }^{8}$ Olivier Chazouilleres ${ }_{1}{ }^{9}$ Christophe Corpechot, ${ }^{9}$ \\ Bruno Fautrel, ${ }^{6}$ Arsène Mekinian, ${ }^{10}$ Elodie Regnier, ${ }^{5}$ David Saadoun, ${ }_{1}^{1,2,3}$ \\ Joe-Elie Salem, ${ }^{11}$ Jérémie Sellam, ${ }^{8}$ Philippe Seksik, ${ }^{7}$ Anne Daguenel-Nguyen, ${ }^{12}$ \\ Valérie Doppler, Jéremie Mariau, ${ }^{4}$ Eric Vicaut, ${ }^{13}$ David Klatzmann ${ }^{1,2}$
}

\section{ABSTRACT}

Objective Regulatory T cells (Tregs) prevent autoimmunity and control inflammation. Consequently, any autoimmune or inflammatory disease reveals a Treg insufficiency. As low-dose interleukin-2 (Id-IL2) expands and activates Tregs, it has a broad therapeutic potential. Aim We aimed to assess this potential and select diseases for further clinical development by crossinvestigating the effects of Id-IL2 in a single clinical trial treating patients with 1 of 11 autoimmune diseases.

Correspondence to

Professor David Klatzmann,

Clinical Investigation Center for Biotherapies and InflammationImmunopathology-Biotherapy department (i2B), Hôpital

Pitié-Salpêtrière, Paris F-75013, France;

david.klatzmann@sorbonneuniversite.fr

$M R$ and $R L$ contributed equally.
Methods We performed a prospective, open-

label, phase I-Ila study in 46 patients with a mild to moderate form of either rheumatoid arthritis, ankylosing spondylitis, systemic lupus erythematosus, psoriasis, Behcet's disease, granulomatosis with polyangiitis, Takayasu's disease, Crohn's disease, ulcerative colitis, autoimmune hepatitis and sclerosing cholangitis. They all received Id-IL2 (1 million IU/day) for 5 days, followed by fortnightly injections for 6 months. Patients were evaluated by deep immunomonitoring and clinical evaluation.

Results Id-IL2 was well tolerated whatever the disease and the concomitant treatments. Thorough supervised and unsupervised immunomonitoring demonstrated specific Treg expansion and activation in all patients, without effector T cell activation. Indication of potential clinical efficacy was observed.

Conclusion The dose of IL-2 and treatment scheme used selectively activate and expand Tregs and are safe across different diseases and concomitant treatments. This and preliminary indications of clinical efficacy should licence the launch of phase II efficacy trial of Id-IL2 in various autoimmune and inflammatory diseases.

Trial registration number NCT01988506.

\section{INTRODUCTION}

Regulatory $\mathrm{T}$ cells (Tregs) prevent autoimmunity and control inflammation. ${ }^{1}$ Consequently, any autoimmune or inflammatory disease denotes a Treg insufficiency. Low-dose interleukin-2 (ld-IL2) expands and activates Tregs, and so has a broad therapeutic potential. ${ }^{2}$ This potential is further supported by the central role of IL-2/IL-2 receptor

\section{Key messages}

What is already known about this subject?

-Whereas high dose interleukin-2 (IL-2) activates effector T cells, low-dose interleukin-2 (Id-IL2) expands and activates regulatory T cells (Tregs), and likewise has a broad therapeutic potential for many autoimmune and inflammatory diseases.

- Proof-of-concept clinical trials have already reported the safety and indication of Id-IL2 efficacy in hepatitis C-related vasculitis, systemic lupus erythematosus and graft versus host disease.

\section{What does this study add?}

- This is the first prospective, phase lla clinical trial that cross-analyses the safety, biological and clinical effects of Id-IL2 across 11 individual diseases chosen to represent the whole spectrum of autoimmune/inflammatory chronic diseases.

- We report that Id-IL2 selectively activates and expands Tregs without activating effector T cells whatever the disease.

- We report signals of efficacy without safety issues of Id-IL2 across diseases by using a unique global evaluation scale, validated in the assessment of psychiatric disease therapies but not yet used in autoimmune/inflammatory diseases.

in autoimmune diseases (AIDs), as recently highlighted in a genetic meta-analysis, ${ }^{3}$ and by IL-2 pleiotropic functions. ${ }^{2}$ Indeed, robust data demonstrate that IL-2 expands Tregs and blocks the differentiation of CD4 naïve T cells into follicular helper or proinflammatory helper 17 (Th17) T cells. ${ }^{4}$ Therefore, ld-IL2 can act on three distinct arms of the immune response involved in AID pathophysiology: cellular and humoral immune responses and inflammation. Likewise, ld-IL2 is now being investigated in various clinical settings. ${ }^{6-12}$ Results of 
How might this impact on clinical practice or future developments?

- Our study highlights a 'universal' biological efficacy and a potential clinical efficacy and safety of Id-IL2 across a wide range of patients suffering from autoimmune/inflammatory conditions.

- These results should licence the initiation of randomised controlled trials in numerous indications in order to confirm these promising preliminary results.

open trials have already yielded promising signs of efficacy, such as in systemic lupus erythematosus (SLE). ${ }^{13} 14$

However, ld-IL2 efficacy may be affected by different factors: (1) Tregs from all patients may not respond similarly to ld-IL2, as some diseases may carry some intrinsic deficit of the IL-2 activation pathway ${ }^{15-18}$; (2) Treg efficacy might be limited by high levels of inflammation such as during flares; (3) the existence of Tregs with appropriate $\mathrm{T}$ cell receptor antigen specificity that could be mobilised for therapy for each disease context is unknown; and (4) the global effect of ld-IL2 may be affected by the fact that Treg-dependent suppression of immune responses and inflammation depends on numerous cells, molecules and pathways that are likely to be affected differently in various AIDs. Finally, although 1d-IL2 activates Tregs at doses at least 20-fold lower than for activating other cell types, ${ }^{12}{ }^{19} 20$ IL2 can affect effector $\mathrm{T}$ cells (Teffs), natural killer cells, type 2 innate lymphoid cells and eosinophils in a (high) dose-dependent manner. ${ }^{21-23}$ Thus, it remains to be seen whether a common appropriate dose/scheme of administration of ld-IL2 can be applied to various AIDs.

To address these questions, we designed a clinical trial in which we treated similarly ld-IL2 patients with 1 of 11 selected AIDs chosen to represent diverse pathophysiological processes. All patients received the same treatment and were monitored similarly. The aim was to cross-analyse the biological and clinical effects of ld-IL2 in heterogeneous patients, such as to appreciate the universality of ld-IL2 effects, and select diseases for conducting further phase II trials. We report here the results of the cross-analysis of 46 treated patients.

\section{METHODS}

\section{Study design and participants}

TRANSREG is a multicentre, interventional open study comparing biochemical and clinical responses to the administration of ld-IL2 across 11 selected diseases (ClinicalTrials.gov trial registration number, NCT01988506). The selected diseases were rheumatoid arthritis (RA), ankylosing spondylitis (AS), SLE, psoriasis, Behçet's disease, granulomatosis with polyangiitis, Takayasu's disease, Crohn's disease (CD), ulcerative colitis (UC), autoimmune hepatitis and sclerosing cholangitis. Patients were selected based on common and disease-specific exclusion and inclusion criteria (online supplementary table S1A and S1B). The main inclusion criteria were a documented diagnosis of at least one of the selected diseases of mild to moderate activity, and being on stable standard therapy for $\geq 2$ months at the time of inclusion. The main exclusion criteria were having another severe or progressive autoimmune/inflammatory disease, haematological disorders, vital organ failure, cancer, and active HIV, Hepatitis B Virus (HBV) or Epstein-Barr Virus (EBV) infections (online supplementary table S1A).
For homogeneity and proper cross-analyses, we report here the results of the first 46 patients who have been treated with IL-2 as Aldesleukin (Proleukin 18 MIU, Novartis Pharma SAS, Rueil-Malmaison, France), before we switched to a different formulation of IL-2 (ILT-101, ILTOO Pharma, Paris, France). Indeed, the use of Proleukin necessitates a cumbersome preparation by a pharmacist to dilute the product and prepare syringes that have limited time span.

\section{Treatment}

We previously showed the dose relation between IL-2 and Treg activation/expansion. ${ }^{8}{ }^{12}$ We selected the dose and scheme of administration of IL-2 used in TRANSREG from these results and a mathematical modelling of the long-term effects of IL-2 administration. ${ }^{24}$ Likewise, patients received 1 Million International Units (MIU)/day of IL-2 from day 1 to day 5 (the induction period), and then every 2 weeks from day 15 to day 180 (the maintenance period). A follow-up visit was made 2 months after the end of the IL-2 treatment (day 240). Patients continued to receive their standard background therapy.

\section{Immunomonitoring}

All the immunomonitoring procedures are described in the online supplementary methods.

\section{Endpoints}

The primary endpoint was the change in the relative concentration of peripheral blood Tregs on day 8 compared with baseline.

Biological secondary endpoints were the area under the curve (AUC) of the changes from baseline in relative concentration of Tregs during the maintenance period from day 30 to day 183 and the changes in inflammation markers from baseline to the end of treatment.

Clinical secondary endpoints were Clinical Global Impression (CGI) ${ }^{25}$ disease-specific and Five-level EuroQol Five-dimensional (EuroQL-5D-5L) scores. Chronic fatigue and arthralgia were also evaluated by physicians at baseline, month 3 and month 6 as they are the most common symptoms shared by patients across all pathologies included in this trial. All clinical evaluation procedures are described in the online supplementary methods.

\section{Statistical analysis}

Changes in Tregs, immunological parameters and inflammation biomarkers between day 1 and day 8 were analysed using analysis of variance for ranked data (Conover's method) considering factor time and disease. The global effect of treatment at the initiation of treatment and its persistence during the maintenance phase was evidenced by demonstrating that the AUCs (calculated by the trapezoidal method) of the changes from baseline between day 1 and day $15\left(\mathrm{iAUC}_{\mathrm{D} 1-\mathrm{D} 15}\right)$ and between day 30 and day $180\left(\mathrm{mAUC}_{\mathrm{D} 30-\mathrm{D} 180}\right)$ were significantly different from zero using Wilcoxon test.

Changes in specific clinical scores and EuroQL-5D-5L were analysed by means of the Wilcoxon test. Changes in CGI severity and activity were analysed by t-test. Fatigue and arthralgia at months 3 and 6 were compared with baseline using Fisher's test.

\section{RESULTS}

Fifty-one patients were included between January 2014 and May 2016. Five patients were not eligible, three because of a viral load of EBV greater than that permitted and two for intercurrent diseases. Thus, 46 patients were treated (figure 1). Patients 


\section{A Trial profile}

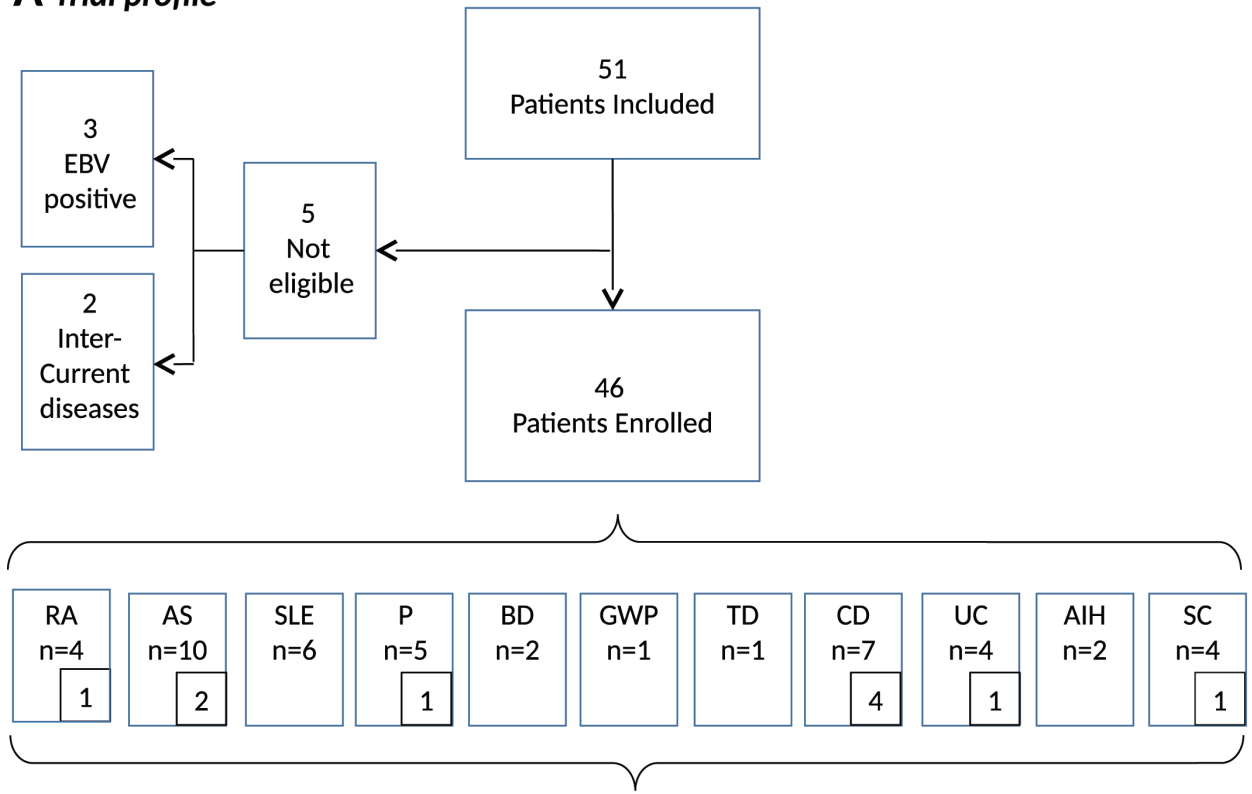

\section{B Study design}

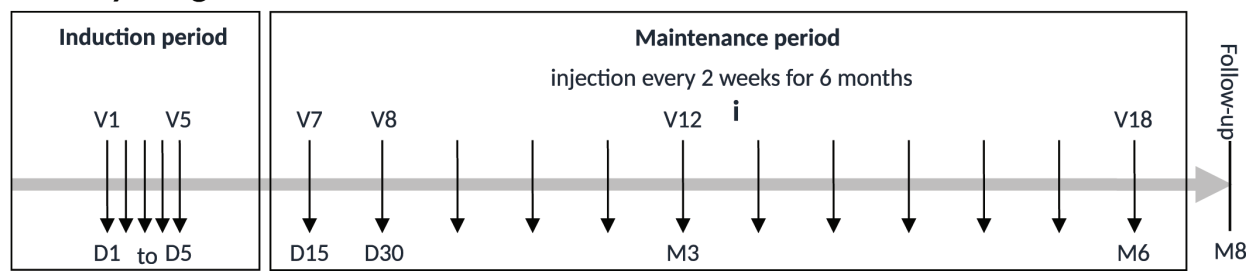

Figure 1 (A) Trial profile. We included 51 patients suffering from 11 autoimmune diseases: rheumatoid arthritis (RA), ankylosing spondylitis (AS), systemic lupus erythematosus (SLE), psoriasis (P), Behcet's disease (BD), granulomatosis with polyangiitis (GWP), Takayasu's disease (TD), Crohn's disease (CD), ulcerative colitis (UC), autoimmune hepatitis (AIH) and sclerosing cholangitis (SC). Five patients were not eligible: three patients for an EBV viral load $>1000$ copies $/ \mathrm{mL}$ and two patients for intercurrent diseases; 10 patients dropped out of the study ( $\mathrm{n}$ in small inserts). (B) Study design. Patients received 1 MIU/day of interleukin-2 from D1 to D5 (the induction period), and then every 2 weeks from D15 to D180 (the maintenance period). D1, day 1; D5, day 5; D15, day 15; D30, day 30; EBV, Epstein-Barr Virus; M3, month 3; M6, month 6; M8, month 8; MIU, Million Intenational Units; $V$, visit.

were heterogeneous in terms of age (23-75 years), disease duration (10-536 months), body mass index (18.3-40.8), per cent of Tregs at baseline $(2.2 \%-12.8 \%)$ and background therapy (online supplementary table S2). Several patients had other concomitant autoimmune or allergic diseases (online supplementary table S2). Concomitant Sjogren's syndrome $(n=3)$, antiphospholipid antibody syndrome $(n=3)$, morphea $(n=1)$, Raynaud's phenomenon $(n=1)$ or psoriasis $(n=1)$ were observed in patients with SLE and RA. Concomitant allergic rhinitis $(n=1)$, allergic asthma $(n=1)$, multiple food allergy $(n=1)$ and cutaneous contact hypersensitivity $(n=1)$ were observed in patients with UC and psoriasis. In agreement with the inclusion criteria, the value of CGI activity and severity score ranged from 0 to 4 at baseline, with mild to moderate specific clinical scores for each disease. There were no other noticeable aspects of baseline demographic and laboratory characteristics of patients.

No major deviations were observed during the study. The most common minor protocol deviations were out of windows visits $(n=11)$ or drug administration not performed because of intercurrent diseases $(n=7)$ in the maintenance period. Ten patients dropped out of the study (online supplementary table S3).

The mean \pm SD baseline percentage of Tregs in patients was $5.8 \% \pm 2.3 \%$ of $\mathrm{CD}^{+} \mathrm{T}$ cells (online supplementary table S2). On day 8 , the primary efficacy endpoint was reached with an increase of Tregs to a mean of $11.1 \% \pm 4.6 \%$, corresponding to a $2.0 \pm 0.6$-fold increase $(\mathrm{p}<0.0001)$ (figure $2 \mathrm{~A}$ and online supplementary table S4A). Treg expansion on day 8 appeared similar across the various diseases for which a minimum of four patients were treated (figure $2 \mathrm{~B}$ ), and cross-comparisons of these responses between diseases showed no significant differences (online supplementary table S4B). Moreover, we did not observe difference in Treg increase between patients receiving antiproliferative drugs and patients receiving non-steroid anti-inflammatory treatments or corticosteroids (online supplementary figure S1). On day 15, that is, 10 days after the last ld-IL2 administration of the induction phase, Treg increase was still significant $(p=0.02)$ (figure $2 \mathrm{~A}$ and online supplementary table $\mathrm{S} 4 \mathrm{~A}$ ). Treg expansion persisted during the maintenance period. From months 1-6, the mean AUC value of Treg changes from baseline for all patients was significantly different from 0 (AUC $_{\mathrm{M} 1-}$ $\left.{ }_{\mathrm{M} 6}=35.1 \pm 13.1, \mathrm{p}<0.001\right)$. It is noteworthy that the recorded results may underestimate the true effect of ld-IL2 because Treg measurements were performed just before the IL-2 injections and thus capture only the residual increase from the previous injection 14 days earlier. The significant changes in percentages of Tregs were also observed for absolute numbers of Tregs (online supplementary table S4A).

There were no detectable effects of ld-IL2 on Teffs (defined as all Foxp $3^{-} \mathrm{CD} 4^{+}$and $\mathrm{CD} 8^{+}$cells), or on activated $\mathrm{CD} 4^{+} \mathrm{C}-$ $\mathrm{D} 25^{\mathrm{lo} /+} \mathrm{Foxp}^{-}$Teffs (online supplementary figure S2). This led 

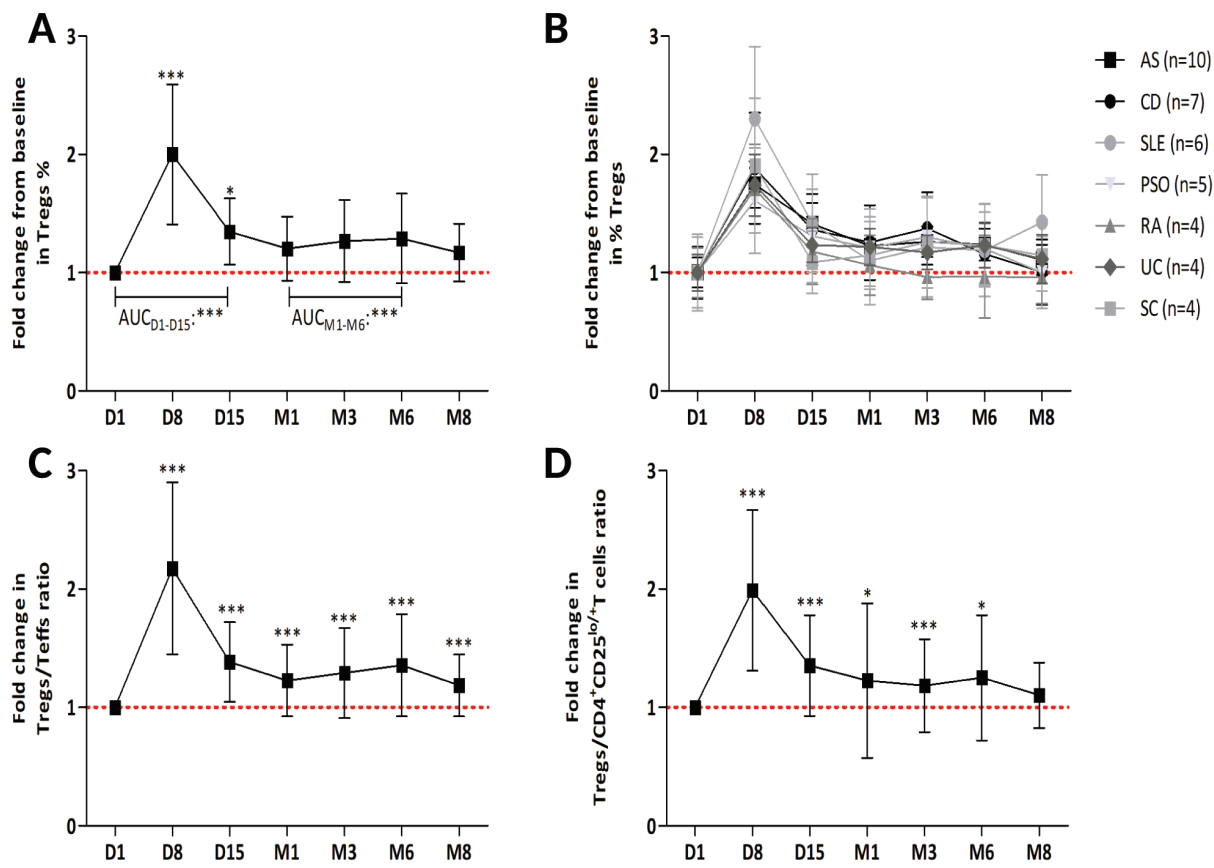

Figure 2 Changes in Treg cells and Teffs cells in patients treated with Id-IL2. Treg cells were gated in CD4 ${ }^{+}$T cells and identified as CD25 ${ }^{\text {hi }}$ CD127 $7^{\text {lo/- }}$ Foxp3 ${ }^{+}$cells. (A) Data represent changes in Tregs as percentages among CD4 ${ }^{+} \mathrm{T}$ cells for all patients from day 1 to month 8 . (B) Data represent changes in Tregs in patients for diseases with $n \geq 4$. (C) Changes in Treg:Teff ratio defined as the percentage of Tregs divided by the percentage of the non-Treg $C D 4^{+}$and $C D 8^{+} T$ cells. (D) Changes in Treg:activated $C D 4^{+} T$ cells ratio as the percentage of Tregs divided by the percentage of $C D 4^{+} C D 25^{10 /+}$ Foxp3 $^{-} \mathrm{T}$ cells. (A-D) Data are represented as mean \pm SD. Data were normalised by baseline values for each patient at the different time points and are represented as fold change, but all statistics were made on raw data. Comparison between day 8 and baseline (main endpoint) was made by Wilcoxon signed-rank test. For each type of cell, global effect of the treatment at its initiation and its persistence during the maintenance phase was evidenced by showing that the AUC of the changes from baseline between day 1 and day 15 (iAUC $C_{D 1-D 15}$ ) and between day 30 and day 180 ( mAUC $_{D 30-D 180}$ ) was significantly different from 0 using Wilcoxon test. ${ }^{*} \mathrm{P}<0.05,{ }^{*} \mathrm{p}<0.01,{ }^{* * *} \mathrm{p}<0.001$. AS, ankylosing spondylitis; AUC, area under the curve; CD, Crohn's disease; D1, day 1; D8, day 8; D15, day 15; Id-IL2, low-dose interleukin-2; M1, month 1; M3, month 3; M6, month 6; M8, month 8; PSO, psoriasis; RA, rheumatoid arthritis; SC, sclerosing cholangitis; SLE, systemic lupus erythematosus; Teffs, effector T cells; Tregs, regulatory T cells; UC, ulcerative colitis.

to a $2.17 \pm 0.72$-fold increase of the Treg:Teff ratio $(\mathrm{p}<0.0001)$ (figure $2 \mathrm{C}$ ), as well as to a $1.98 \pm 0.42$-fold increase of Tregs/ activated non-Treg $\mathrm{CD}^{+}$cells $(p<0.0001)$ at the end of the induction course (figure 2D). It is worth noting that every single patient responded to $1 \mathrm{MIU} /$ day of IL-2 by expanding their percentage of Tregs in the peripheral blood by at least $25 \%$.

Because unwanted stimulation of Teff could be deleterious in the treatment of AIDs, the demonstration of a specific effect of 1d-IL2 on Tregs is of utmost importance for the treatment of AIDs caused by Teffs. Although classic immunophenotyping did not show any expansion of non-Treg $\mathrm{T}$ cells, we further assessed that the effects of IL-2 are Treg-specific in a group of nine patients in whom an extensive immunophenotyping was performed $^{26}$ and using unsupervised analyses. Using t-distributed stochastic neighbor embedding (t-SNE) and flowMeans $\mathrm{R}$ packages, ${ }^{27-29}$ clusters were defined automatically based on Foxp3 and CD127 expression (figure 3A). Cluster 6 corresponded to Foxp $3^{+} \mathrm{CD} 127^{- \text {low }}$ cells and thus defines the Treg cluster, while the Teff cells corresponded to all the other clusters. We repeated the procedure to automatically recluster separately Tregs and Teffs based on the other markers of staining (CD25, Helios, CD45RA and CCR5). This generated six and eight clusters for Tregs and Teffs, respectively (figure 3B,D). For each cluster, comparison of the different values per marker on day 8 versus baseline showed statistically different values only for Tregs $(p=0.03)$, and among them those with the more activated phenotypes (figure 3C). There were no differences for any of the Teff subsets (figure $3 \mathrm{E}$ ), with $\mathrm{p}$ value not even close to significance. The same analysis strategy was then applied to another panel of antibodies (online supplementary figure S3). Here again, only two Treg subsets corresponding to those with the phenotype of either resting/naïve $\mathrm{CD} 45 \mathrm{RA}^{+} \mathrm{CD} 95^{+} \mathrm{CCR} 7^{+}$ $\mathrm{ICOS}^{+/-} \mathrm{HLA}_{-} \mathrm{DR}^{-}$) or activated/memory Tregs $\left(\mathrm{CD} 45 \mathrm{RA}^{-} \mathrm{C}\right.$ D95 ${ }^{+} \mathrm{CCR}^{+/-} \mathrm{ICOS}^{+/-} \mathrm{HLA}^{+} \mathrm{DR}^{+}$) were significantly expanded on day 8 while other $T$ cell subsets were not affected.

Analysis of T, B and natural killer (NK) cell subsets showed little change during the induction period (online supplementary table S4A). As previously described, ${ }^{12}$ we observed an increased frequency of the regulatory CD56 ${ }^{\text {bright }} \mathrm{NK}$ cell subset (online supplementary figure S4A-C). Eosinophils levels were heterogeneous at baseline. For the 43 patients with normal counts, we observed a slight increase on day 8 that stayed under the normal value limit, or slightly above in two patients. For the three patients with eosinophilia at baseline, eosinophil counts approximately doubled after the induction period for two of them and remained stable for the other. All values returned to baseline during the maintenance treatment (online supplementary figure S5).

Plasma levels of Th1/Th2/Th17/Treg cytokines were unchanged all along treatment (online supplementary figure S6). In agreement with the mild to moderate activity of the diseases, C-reactive protein (CRP) was detected $(>5 \mathrm{mg} / \mathrm{dL})$ in only 10 

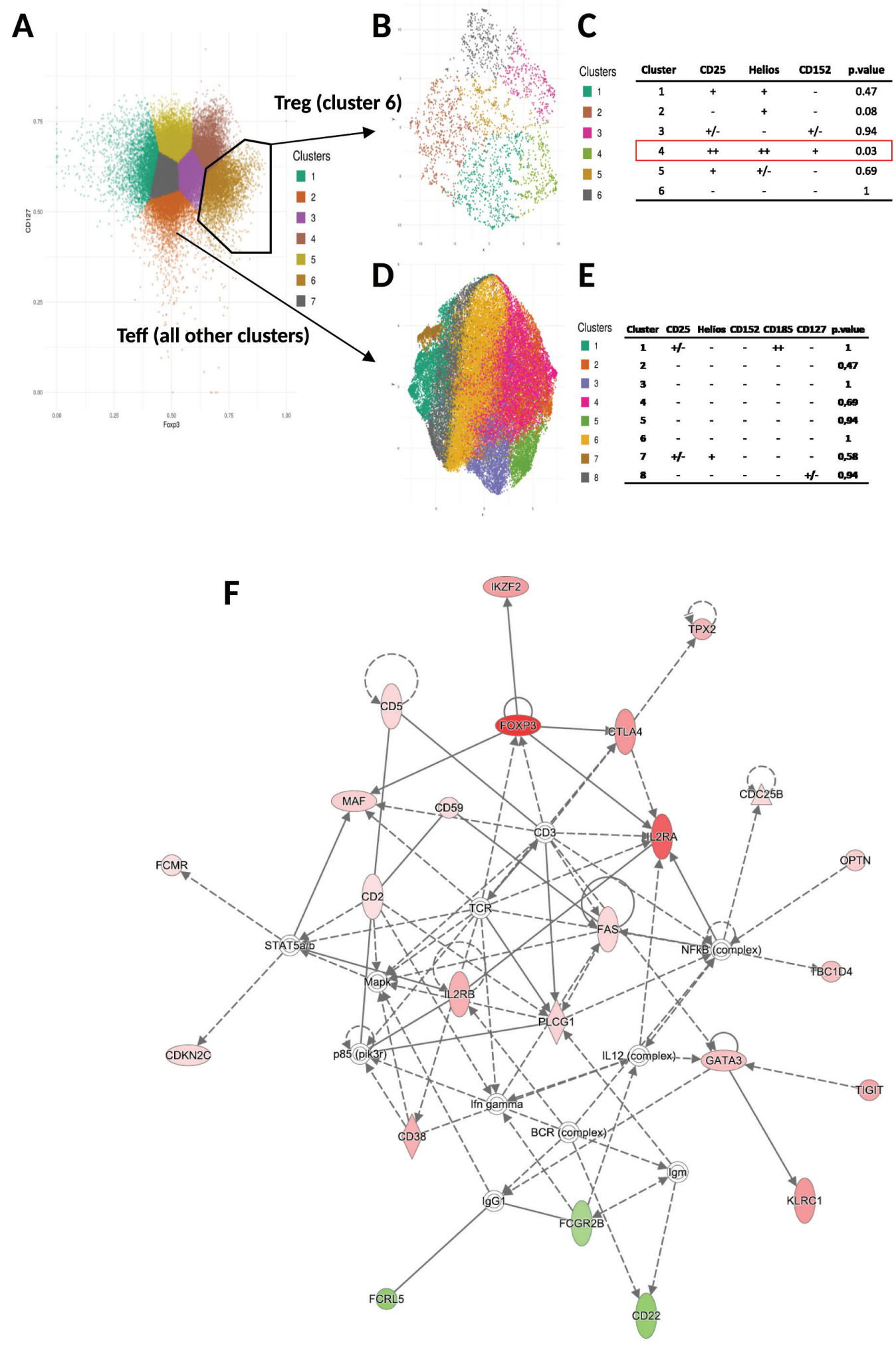

Figure 3 Unsupervised analysis of Treg phenotype and transcriptome. (A-E) Cells were stained for Helios, CD25, CXCR5, Ki67, CTLA-4, F0XP3, CD8, CD127, CD4 and CD8. A first step of automatic clustering based on the expression of FOXP3 and CD127 in CD3 ${ }^{+}$CD4 ${ }^{+}$cells was performed on a merge of samples from baseline and day 8 for the nine patients studied. (A) The algorithm generated seven clusters that are represented in different colours on a FoxP3/CD127 biplot. (B and D) A second step of automatic clustering was performed separately on Tregs (cells from cluster 6 in A) and Teffs (cells from all other clusters in $\mathrm{A}$ ) based on FoxP3 ${ }^{-} \mathrm{CD}^{+} \mathrm{CD}^{+}$cells. ( $\mathrm{C}$ and $\mathrm{E}$ ) $\mathrm{P}$ values of the statistical analysis of the difference between baseline and day 8 for each cluster identified in $B$ and $D$, respectively (statistical test: Wilcoxon pairwise test, $p$ values $<0.05$ were considered as significant). (C) Also shows the phenotype of each cluster of Treg cells. (F) Transcriptomic analysis of significantly regulated genes from PBMCs on day 8 compared with baseline using Ingenuity Pathway Analysis reveals a Treg-related pathway (upregulated genes in red and downregulated genes in green; direct and indirect interactions between molecules are depicted by solid and dotted lines, respectively). PBMCs, peripheral blood mononuclear cells; Teffs, effector T cells; Tregs, regulatory T cells. 
patients at baseline and thus could not be studied as an endpoint (online supplementary figure S7).

We reported that ld-IL2 has a global anti-inflammatory effect based on a transcriptome analysis of peripheral blood mononuclear cells (PBMCs). ${ }^{6}$ Here, we similarly analysed the global transcriptome of PBMCs before and after 1d-IL2. We found 91 differentially expressed genes with a Benjamini-Hochberg corrected p value $<0.05$ on day 8 versus baseline (online supplementary table S5). Ingenuity Pathway Analysis showed significant enrichment of four pathways/signatures all directly or indirectly related to Treg. The most significantly modulated pathway $(-\log (p$ value $)>3)$ is organised around upregulated genes such as Foxp3, IL-2Ra and Rb and CTLA4 that are essential for Treg function (figure $3 \mathrm{~F}$ ). We also assessed the modulation of a recent Treg signature defined from single cell transcriptomic. ${ }^{30}$ This signature was significantly upregulated on day 8 versus baseline $(\mathrm{p}<1 \mathrm{e}-04)$ as well as at month 3 versus baseline $(\mathrm{p}<0.03)$, indicating that ld-IL2 effects on Tregs were maintained over time across diseases (online supplementary figure S8).

As previously reported, we did not observe anti-IL-2 antibodies in patients' plasma after ld-IL2 treatment (online supplementary figure S9). ${ }^{12} 31$

Low-dose recombinant human (rh)IL-2 was well tolerated. Six patients displayed seven serious adverse events, none of which was considered related to IL-2 (online supplementary table S6A). Most non-serious adverse events (NSAEs) were injection site reactions, which occurred in approximately a quarter of the injections. The frequency of seasonal upper or lower respiratory tract infections $(n=28)$, with associated fever of over $38^{\circ} \mathrm{C}$ in 17 of them, was as expected. The investigators did not report any unforeseen outcome of these infections (online supplementary table S6A). Finally, the analysis of the NSAEs according to background therapy did not show any significant difference (online supplementary table S6B).

Clinical secondary endpoints were CGI, disease-specific and EuroQL-5D-5L scores. CGI was selected as a clinical evaluation method that could work across our heterogeneous group of diseases. Indeed, CGI was originally developed for use in clinical trials to provide a brief, stand-alone assessment of the clinician's view of the patient's global functioning prior to and after initiating a study medication. ${ }^{25}$ CGI is commonly used in psychiatry but has not yet been validated in AIDs. Compared with baseline, a statistically significant improvement of the CGI scored by the physician was found at months 3 and 6 for CGI activity $(\mathrm{p}<0.001)$ (figure $4 \mathrm{~A})$ and at month 6 for CGI severity $(p<0.001)$ (figure 4B). Significant changes were also found at the follow-up visit 2 months after discontinuation of the treatment (CGI activity $p=0.02$ and CGI severity $p=0.04$ ). Among the 46 treated patients, there were 26 with documented arthralgia and 26 with chronic fatigue at baseline. At month 3, there was a significant decrease of the percentage of patients with fatigue $(p=0.002)$ and with arthralgia $(p=0.00015)$ (figure $4 C)$, and this trend continued at month 6 . Evaluation of the impact of ld-IL2 on quality of life using EuroQL-5D-5L showed a non-significant improvement. We also assessed the disease-specific score for diseases with at least four patients treated. There was an improvement for AS (figure 5A), UC (figure 5B), SLE (figure 5C and online supplementary figure $S 9$ ) and psoriasis (figure $5 \mathrm{D}, \mathrm{E}$ ), but not for CD (online supplementary table S7).

\section{DISCUSSION}

We previously reported a double-blind, placebo-controlled dosefinding study of ld-IL2 in type 1 diabetes. ${ }^{8}$ This identified a dose
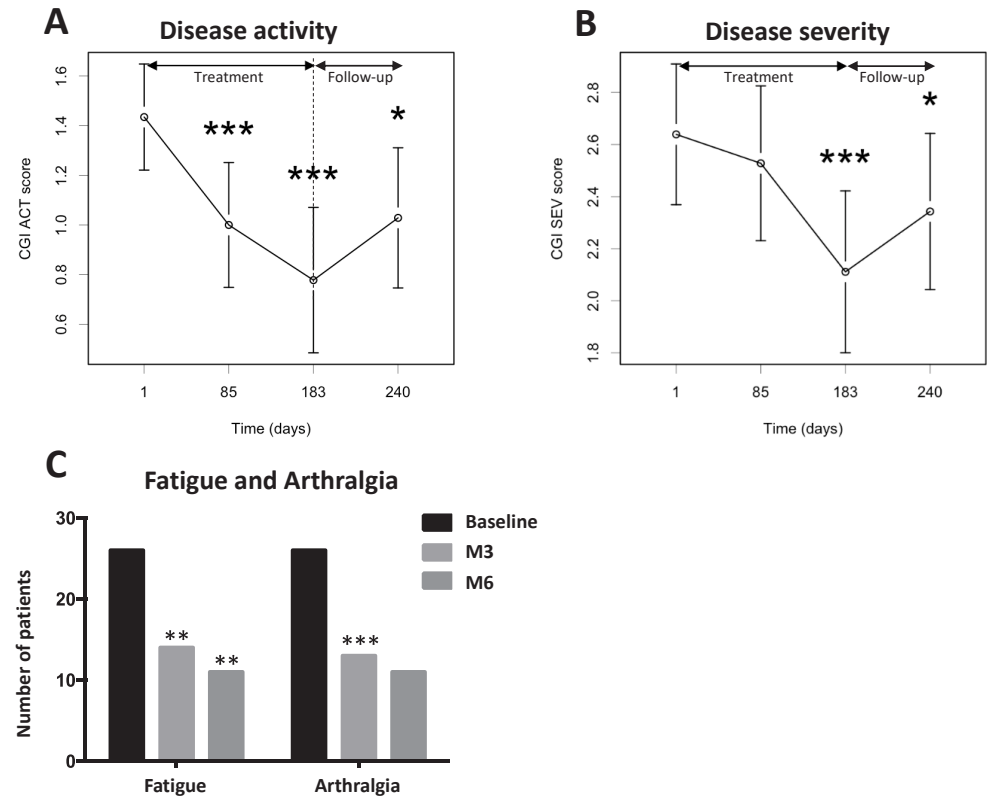

Figure 4 Clinical effects of Id-IL2 across the 11 autoimmune diseases. Clinical Global Impression (CGI) for (A) activity and (B) severity was scored by the physician at baseline (day 1), month 3 (day 85), month 6 (day 183) and at a follow-up visit at month 8 (day 240). Data are represented as mean \pm SD. Data were compared with baseline using t-test. (C) Arthralgia pain intensity and fatigue level were assessed by the physician at baseline, month 3 and month 6 . Data are represented as the number of patients presenting arthralgia or fatigue. Data were compared with baseline using Fisher's test. ${ }^{*} \mathrm{P}<0.05,{ }^{* *} \mathrm{p}<0.01,{ }^{* *} \mathrm{p}<0.001$. ACT, activity; Id-IL2, low-dose interleukin-2; SEV, severity; M3, month 3; M6, month 6 . 

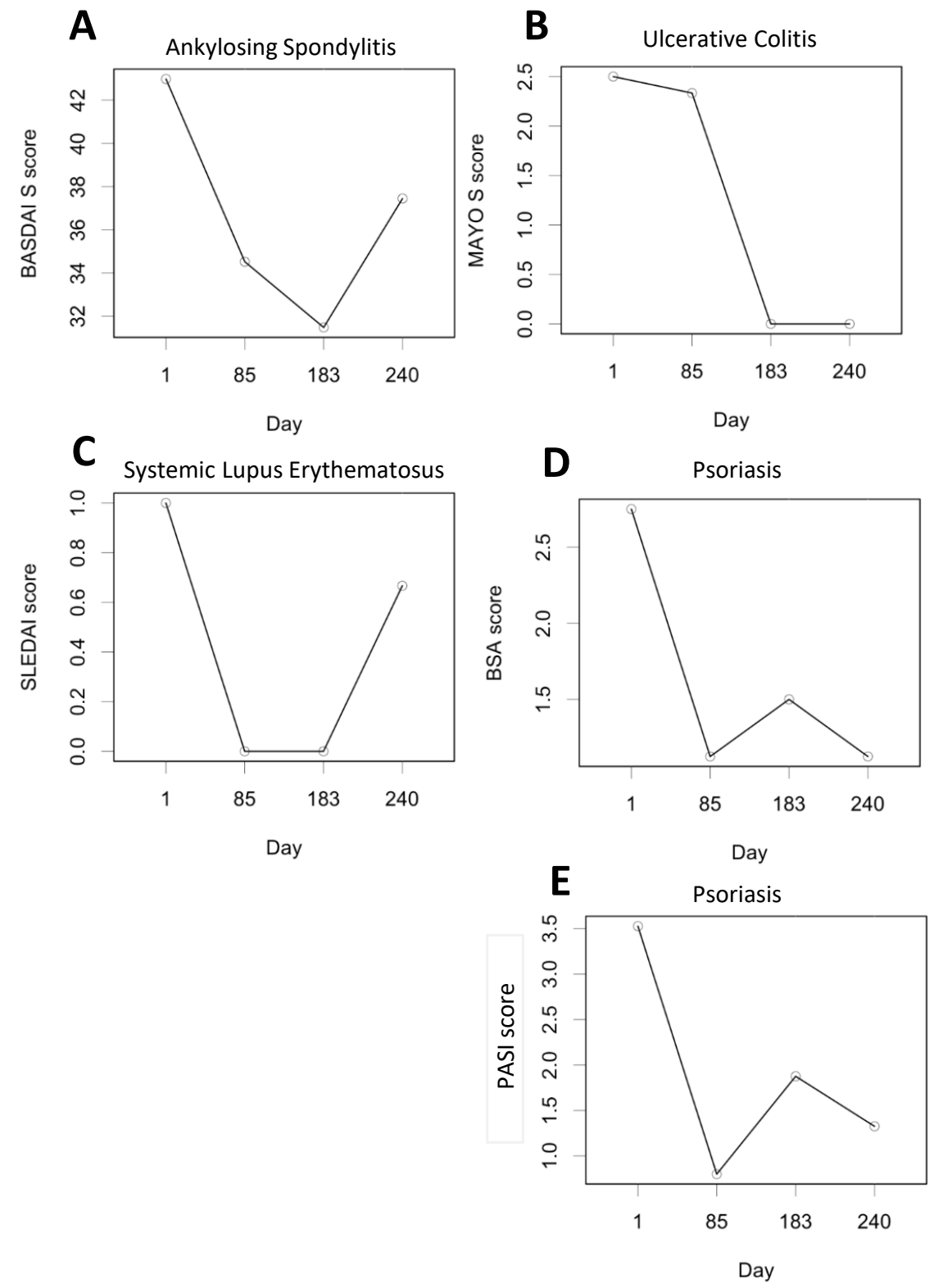

Figure 5 Clinical effects of low-dose interleukin-2 in specific diseases. Specific clinical scores were measured at baseline (day 1), month 3 (day 85 ),

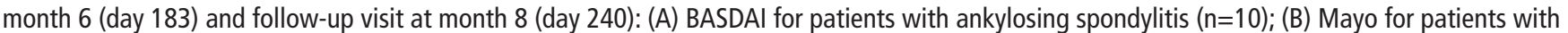
ulcerative colitis $(n=4)$; (C) SLEDAI for patients with systemic lupus erythematosus $(n=6)$; (D) BSA and (E) PASI for patients with psoriasis ( $n=4)$. Data are represented as means. BASDAI, Bath Ankylosing Spondylitis Disease Activity Index; BSA, body surface area; PASI,Psoriasis Area Severity Index; SLEDAI, Systemic Lupus Erythematosus Disease Activity Index.

of $1 \mathrm{MIU} /$ injection as well tolerated and boosting Tregs without effects on Teffs. ${ }^{8} 12$ Given the heterogeneity of ld-IL2 target diseases and of their pathophysiological background, it remained to investigate how 'universal' would be the effects of this dosage. We thus initiated a trial aimed at cross-evaluating ld-IL2 in 11 different AIDs chosen to cover diseases that are organ-specific and systemic, T cell-mediated or antibody-mediated, and with high or little inflammation.

Our supervised analyses clearly demonstrated that $1 \mathrm{MIU} /$ injection, with the scheme used, selectively activates and expands Tregs without activating Teffs, whatever the disease. This translates in a significant increase of both the Treg/Teff as well as the Treg/activated CD4 $+\mathrm{T}$ cell ratios. A similar response was observed for patients with low or high Treg counts at baseline. Both naïve and activated/memory Tregs expanded after ld-IL2, while we did not detect expansion of CD4 effector memory cells. As previously reported, at the dose used, we did not observe an effect on the overall NK cell population, but only on the non-cytotoxic CD56 ${ }^{\text {hi }}$ NK cell subset, also called regulatory NK cells. Given the importance of the specificity of the effect on Tregs, we also evaluated it using unsupervised analyses that can be considered as less biased. This fully confirmed the specificity of the ld-IL2 effects for Tregs, further showing that the only cells responding to ld-IL2 were the resting/naive and activated/memory Tregs. Thus, supervised and unsupervised cellular and molecular analyses indicate that, with the dose/scheme used, ld-IL2 triggers a 'universal' specific effect for Tregs across a group of very heterogeneous patients.

Although this trial was powered only to evaluate the effects of IL-2 on Tregs, we also monitored secondary efficacy criteria relating to clinical status. With the idea of a cross-analysis of 
diseases with various symptoms and scores, we chose CGI as our main per protocol clinical endpoint for cross-evaluation. Despite the fact that patients had mild or moderate disease forms, thus low CGI scores at baseline, and were heterogeneous, we observed an overall significant improvement of CGI scores. Improvements in CGI scores were already noted at month 3 and continued to increase at month 6 . A potential clinical benefit was also evaluated across diseases by specifically monitoring arthralgia and chronic fatigue, which were the most shared symptoms of our patients. There was a significant improvement of these symptoms, already noted at month 3 . Two months after treatment discontinuation, CGI scores had a tendency to increase but were still significantly improved compared with baseline. No flare was observed during this period. Finally, we evaluated the disease-specific scores for diseases with such available scores and with at least four patients included. As previously reported, we saw improvement in SLE. We also saw improvements for patients with UC, AS and psoriasis but not in those with CD. Altogether, these evaluations converge to suggest a broad potential of ld-IL2 and contributed to the selection of SLE as the target disease of an ongoing phase II trial (NCT02955615). They also suggest to further evaluate CGI in the field of AIDs. Indeed, as many drugs being developed target pathways involved in multiple AIDs, they thus have a broad therapeutic potential. Our trial design and methods could represent an early clinical cross-evaluation step that would help select diseases for further evaluation.

The safety profile of 1d-IL2 across the different diseases and across various background treatments was very good. There has been no serious adverse event related to treatment. The most frequent adverse events were reaction at the injection sites, which are common for biologics, and are of unknown mechanisms. As in other trials of ld-IL2, we did not observe induction of antiIL-2 antibodies under treatment. ${ }^{12} 31$ Based on safety data from our previous clinical trials and our modelling of the effects of IL-2 on Tregs, ${ }^{24}$ we used in this trial the dose of $1 \mathrm{MIU} /$ injection, with once-a-fortnight injections during the maintenance course. Should a more pronounced effect on Treg during the maintenance phase be desired, one could use weekly injections as we do in our current LUPIL-2 trial (NCT02955615).

Altogether, our study highlights the 'universal' safety, biological efficacy and possible clinical efficacy of ld-IL2 across a group of very heterogeneous patients. It also highlights that the therapeutic window of plain IL-2 is satisfactory and thus licences the initiation of phase II efficacy trials, which are now necessary to ascertain the therapeutic potential of ld-IL2.

\footnotetext{
Author affiliations

'Inflammation-Immunopathology-Biotherapy Department (i2B), AP-HP, PitiéSalpêtrière Hospital, Paris, France

${ }^{2}$ Immunology-Immunopathology- Immunotherapy (i3), Sorbonne Université, INSERM, Paris, France

${ }^{3}$ Department of Internal Medicine and Clinical Immunology, AP-HP, Pitié-Salpêtrière Hospital, Paris, France

${ }^{4}$ ILTOO Pharma, Paris, France

${ }^{5}$ Department of Dermatology, AP-HP Cochin Hospital, Paris, France

${ }^{6}$ Department of Rheumatology, AP-HP Pitié-Salpêtrière Hospital, Paris, France

${ }^{7}$ Department of Gastroenterology, AP-HP Saint Antoine Hospital, Paris, France

${ }^{8}$ Department of Rheumatology, AP-HP Saint-Antoine Hospital, Paris, France

${ }^{9}$ Department of Hepatology, AP-HP Saint-Antoine Hospital, Paris, France

${ }^{10}$ Department of Internal Medicine, AP-HP, Saint-Antoine Hospital, Paris, France

${ }^{11}$ Department of Pharmacology and CIC-1421, AP-HP Pitié-Salpêtrière Hospital, Paris, France

${ }^{12}$ Department of Pharmacy, AP-HP Saint-Antoine Hospital, Paris, France

${ }^{13}$ Unité de Recherche Clinique and Université Paris, AP-HP Saint Louis/Lariboisière Hospitals, Paris, France
}

Acknowledgements We thank the patients and the members of the TRANSREG study group for their participation. We thank all the personnel of the Clinical
Investigation Center Paris-Est (CIC-PE), in particular C Funck-Brentano, E Dasque, $\mathrm{L}$ Koehl and $\mathrm{M}$ Chenier, and the personnel of the Clinical Investigation Center for Biotherapies (CIC-BTi), Guillaume Churlaud, Michèle Barbié, Cornélia Degbé, Natalie Féry, Anne Laure Raveu and Alexandra Roux, for their excellent help. We thank G Bensimon for his initial contribution to the trial design and the selection of diseases. We also thank the UMR 8199 LIGAN-PM Genomics platform (Lille, France), which belongs to the 'Federation de Recherche' 3508 LabEx EGID (European Genomic Institute for Diabetes; ANR-10-LABX-46) and was supported by the ANR Equipex 2010 session (ANR-10-EQPX-07-01; 'LIGAN-PM'). The LIGAN-PM Genomics platform (Lille, France) is also supported by the FEDER and the Region Nord-Pas-deCalais-Picardie.

Collaborators Principal Investigator: Prof D Klatzmann MD, PhD. Methodologist: Prof E Vicaut, MD, PhD. Clinical Investigator: R Lorenzon MD. Clinical pathologist: M Rosenzwajg MD, PhD. Clinical Investigations Centers: (1) Clinical Investigation Center Paris-Est CIC-1421 Pitié-Salpêtrière Paris France: Prof C. Funck Brentano MD, PhD; J-E Salem, MD, PhD; E. Guilloux, (2)Clinical Investigation Center for Biotherapy and immunology CIC-1420 Pitié-Salpêtrière Paris France: M Barbie, N Fery, C Degbe, C Bernard, C Ribet. Clinicians and recruiting centers: (1) Prof $S$ Aractingi MD, PhD; E Regnier, MD: Department of Dermatology, AP-HP, Cochin Hospital (2) Prof L Beaugerie MD, PhD; Prof P. Seksik: Department of Gastroenterology, AP-HP, Saint Antoine Hospital (3) Prof F Berenbaum MD, PhD; Prof J. Sellam MD, PhD: Department of Rheumatology, APHP, Saint-Antoine Hospital (4) Prof P Cacoub MD, PhD: Department of Internal Medicine, AP-HP, PitiéSalpêtrière Hospital (5) Prof O Chazouilleres MD, PhD, Dr C. Corpechot MD, PhD: Department of Hepatology APHP, Saint Antoine Hospital (6) Prof B Fautrel MD, PhD: Department of Rheumatology, AP-HP, Pitié-Salpêtrière Hospital (7) Dr A Mekinian, MD, PhD; Pr Fain, MD: Department of Internal Medicine, AP-HP, Saint Antoine Hospital. Treatment Management: Pharmacy, Saint Antoine Hospital, APHP, Paris, France: C Mayala PharmD; A. Daguenel Nguyen PharmD, V Quiniou Pharm D, PA Vinot Pharm D; Pharmacy, Pitié Salpêtrière Hospital APHP, Paris, France: Fanny Charbonnier-Beaupel. Regulatory affairs and Monitoring: Clinical Research and Development Department (DRCD) APHP, Paris, France: K. Seymour-Inamo; Clinical Research Unit Lariboisière, APHP, Paris, France: E. Vicaut MD, PhD; V. Jouis, A. Mekouo-Tagne, O. Tran. Data and Safety Monitoring Board: (1) Prof Jacques Eric Gottenberg: Department of Rheumatology, Hautepierre Hospital, Strasbourg (2) Prof Olivier Blétry: Department of Internal Medicine, Foch Hospital, Suresnes (3) Prof Pierre Galanaud: Department of Internal Medicine and Clinical Immunology, Antoine Béclère Hospital, Clamart.

Contributors MR participated in the design of the study, supervised immunomonitoring, analysed the results and helped write the article. RL participated in the design of the study, was the principal clinical investigator, analysed the results and helped write the article. PC participated in the design of the study and was a clinical investigator of the study. FP, KES and HPP analysed the immunological and transcriptomic results. SA, BB, LB, FB, BF, JC, OC, CC, CF, AM, ER, DS, PS, JS and J-ES were clinical investigators of the study. $C R$ was an assistant clinical investigator and supervised the logistics. CB and AD-N were in charge of treatment management. VD and JM contributed to the study performance and regulatory affair follow-up. EV participated in the design of the study, wrote the statistical report, analysed the results and helped write the article. DK conceived and supervised the study, was the principal investigator, analysed the results and wrote the first draft of the article. All authors edited and approved the final draft of the article.

Funding Funding was provided by the sponsor, the Assistance Publique - Hôpitaux de Paris. ILTOO Pharma provided support to the sponsor for clinical trial conduct and regulatory affairs, and contributed to some statistical analyses. Additional funding came from the ANR within the Investissements d'Avenir programme (ANR-11IDEX-0004-02, LabEx Transimmunom), the Recherche Hospitalo-Universitaire (ANR16-RHUS-0001, RHU IMAP) and the European Research Council Advanced Grant (FP7-IDEAS-ERC-322856, TRiPoD).

Competing interests $M R, R L, P C, F B, B F, P C, J S, D S, C B$ and $D K$ are inventors for patent applications related to the therapeutic use of Id-IL2, which belongs to their academic institutions and has been licensed to ILTOO Pharma. MR, VD, JM and DK hold shares in ILTOO Pharma. HPP, VD and JM are employees of ILTOO Pharma. No other potential conflicts of interest relevant to this article were reported.

Patient consent Obtained.

Ethics approval The study was approved by the institutional review board of PitiéSalpêtrière Hospital (CPP-IdFVI) and performed in accordance with the Declaration of Helsinki and good clinical practice.

Provenance and peer review Not commissioned; externally peer reviewed.

\section{REFERENCES}

1 Wing K, Sakaguchi S. Regulatory T cells exert checks and balances on self tolerance and autoimmunity. Nat Immunol 2010;11:7-13.

2 Klatzmann D, Abbas AK. The promise of low-dose interleukin-2 therapy for autoimmune and inflammatory diseases. Nat Rev Immunol 2015;15:283-94. 
3 Li YR, Li J, Zhao SD, et al. Meta-analysis of shared genetic architecture across ten pediatric autoimmune diseases. Nat Med 2015;21:1018-27.

4 Laurence A, Tato CM, Davidson TS, et al. Interleukin-2 signaling via STAT5 constrains T helper 17 cell generation. Immunity 2007;26:371-81.

5 Ballesteros-Tato A, León B, Graf BA, et al. Interleukin-2 inhibits germinal center formation by limiting $T$ follicular helper cell differentiation. Immunity 2012;36:847-56.

6 Saadoun D, Rosenzwajg M, Joly F, et al. Regulatory T-cell responses to low-dose interleukin-2 in HCV-induced vasculitis. N Eng/ J Med 2011;365:2067-77.

7 Koreth J, Matsuoka K, Kim HT, et al. Interleukin-2 and regulatory T cells in graftversus-host disease. N Engl J Med 2011;365:2055-66.

8 Hartemann A, Bensimon G, Payan CA, et al. Low-dose interleukin 2 in patients with type 1 diabetes: a phase 1/2 randomised, double-blind, placebo-controlled trial. Lancet Diabetes Endocrinol 2013;1:295-305.

9 Castela E, Le Duff F, Butori C, et al. Effects of low-dose recombinant interleukin 2 to promote T-regulatory cells in alopecia areata. JAMA Dermatol 2014;150:748-51.

10 Kennedy-Nasser AA, Ku S, Castillo-Caro P, et al. Ultra low-dose IL-2 for GVHD prophylaxis after allogeneic hematopoietic stem cell transplantation mediates expansion of regulatory $T$ cells without diminishing antiviral and antileukemic activity. Clin Cancer Res 2014:20:2215-25.

11 Koreth J, Ritz J, Tsokos GC, et al. Low-dose Interleukin-2 in the Treatment of Autoimmune Disease. Oncology \& Hematology Review 2014;10:157-63.

12 Rosenzwajg M, Churlaud G, Mallone R, et al. Low-dose interleukin-2 fosters a dose-dependent regulatory T cell tuned milieu in T1D patients. J Autoimmun 2015;58:48-58.

13 He J, Zhang $X$, Wei Y, et al. Low-dose interleukin-2 treatment selectively modulates CD4(+) T cell subsets in patients with systemic lupus erythematosus. Nat Med 2016;22:991-3.

14 von Spee-Mayer C, Siegert E, Abdirama D, et al. Low-dose interleukin-2 selectively corrects regulatory T cell defects in patients with systemic lupus erythematosus. Ann Rheum Dis 2016;75:1407-15.

15 Zier KS, Leo MM, Spielman RS, et al. Decreased synthesis of interleukin-2 (IL-2) in insulin-dependent diabetes mellitus. Diabetes 1984;33:552-5.

16 Kitas GD, Salmon M, Farr M, et al. Deficient interleukin 2 production in rheumatoid arthritis: association with active disease and systemic complications. Clin Exp Immunol 1988;73:242-9.

17 Lieberman LA, Tsokos GC. The IL-2 defect in systemic lupus erythematosus disease has an expansive effect on host immunity. J Biomed Biotechnol 2010;2010:1-6.
18 Comte D, Karampetsou MP, Kis-Toth K, et al. Brief Report: CD4+ T cells from patients with systemic lupus erythematosus respond poorly to exogenous interleukin-2. Arthritis Rheumatol 2017;69:808-13.

19 Yu A, Snowhite I, Vendrame F, et al. Selective IL-2 responsiveness of regulatory T cells through multiple intrinsic mechanisms supports the use of low-dose IL-2 therapy in type 1 diabetes. Diabetes 2015;64:2172-83.

20 Matsuoka K, Koreth J, Kim HT, et al. Low-dose interleukin-2 therapy restores regulatory $\mathrm{T}$ cell homeostasis in patients with chronic graft-versus-host disease. Sci Transl Med 2013;5:179ra43.

21 Ito S, Bollard CM, Carlsten M, et al. Ultra-low dose interleukin-2 promotes immunemodulating function of regulatory T cells and natural killer cells in healthy volunteers. Mol Ther 2014;22:1388-95.

22 Liao W, Lin JX, Leonard WJ. Interleukin-2 at the crossroads of effector responses, tolerance, and immunotherapy. Immunity 2013;38:13-25.

23 Van Gool F, Molofsky AB, Morar MM, et al. Interleukin-5-producing group 2 innate lymphoid cells control eosinophilia induced by interleukin-2 therapy. Blood 2014;124:3572-6.

24 Churlaud G, Abbara C, Vinot PA, et al. Pharmacodynamics of regulatory T cells in mice and humans treated with low-dose IL-2. J Allergy Clin Immunol 2018;142:1344-6.

25 Busner J, Targum SD. The clinical global impressions scale. Psychiatry Edgmont 2007:4:28-37.

26 Pitoiset F, Cassard L, El Soufi K, et al. Deep phenotyping of immune cell populations by optimized and standardized flow cytometry analyses. Cytometry $A$ 2018;93:793-802.

27 Aghaeepour N. FlowMeans: non-parametric flow cytometry data gating. R Package Version. 2010. Available:http://bioconductor.jp/packages/3.1/bioc/vignettes/ flowMeans/inst/doc/flowMeans.pdf

28 der MLvan, Hinton G. Visualizing data using t-SNE. J Mach Learn Res 2008;9:2579-605.

29 Van Der Maaten L. Accelerating t-SNE using tree-based algorithms. J Mach Learn Res 2014; 15:3221-45

30 De Simone M, Arrigoni A, Rossetti G, et al. Transcriptional landscape of human tissue lymphocytes unveils uniqueness of tumor-infiltrating T regulatory cells. Immunity 2016;45:1135-47.

31 Churlaud G, Rosenzwajg M, Cacoub P, et al. IL-2 antibodies in type 1 diabetes and during IL-2 therapy. Diabetologia 2018;61:2066-8. 